\title{
Asymptotic Noise Analysis of Time Reversal Detection
}

\author{
Yuanwei Jin and José M.F. Moura \\ Department of Electrical and Computer Engineering \\ Carnegie Mellon University \\ Pittsburgh, PA $15213^{*}$
}

\begin{abstract}
In time reversal, the received signal, including the additive noise component, is time reversed, energy normalized, and re-transmitted. The noise contained in the re-transmitted time-reversed signal has a significant impact on the time reversal detection performance. This paper carries out an asymptotic noise analysis of the time reversal generalized likelihood ratio detector, and develops an approximate closed form of the asymptotic SNR gain of the time reversal detector compared with the conventional detector. We show that a rich scattering and low noise environment is preferred for time reversal.
\end{abstract}

\section{Introduction}

In time reversal (phase conjugation in frequency domain), a short pulse, for example, transmitted by a source through a dispersive medium, is received by an array, then time reversed, energy normalized, and retransmitted through the same medium. If the scattering channel is reciprocal and sufficiently rich, the retransmitted waveform refocuses on the original source. In our recent work, [1], we considered the signal detection problem by time reversal using a pair of transmit/receive antennas. Among them, the time reversal generalized likelihood ratio detector (TR-GLRT) is developed, and its performance is compared with the conventional detection.

An important issue in the time reversal detection is the noise effect. Time reversal is performed iteratively. First, a probing signal illuminates the target area by a transmit array, and is received at a receive array. The received signal contains the backscatters from the scattering medium and the additive noise due to the sensing device or other mechanisms. Next, the received total signal, including the noise, is time reversed (or phase conjugated in frequency domain), energy normalized, sent back to the same channel, and recorded at the initial transmit array. Inevitably, part of the transmission power in the second step will be wasted on transmitting the additive noise received in the first step. In other words, the effective output signal-to-noise ratio (SNR)

\footnotetext{
* This work is funded by the Defence Advanced Research Projects Agency through the Army Research Office under grant no. W911NF-04$1-0031$
}

in the second step is reduced due to the inserted noise in the time reversal step.

The goal of this paper is to study the noise impact on the detection performance. We choose a general multi-static transmit/receive array configuration, which is also called Multiple-input Multiple-out (MIMO) in recent radar literature. The two arrays, denoted by A and B, can switch between transmit mode and receive mode. The construction of the TR-GLRT developed in [1] takes two steps, i.e., calculating the maximum likelihood estimate of the unknown target channel response from the measurements, followed by computing the test statistics. To examine the impact of the noise contained in the time reversed signal, we assume that the target channel response is known. This assumption implies an asymptotic analysis of the noise impact - the estimate of the target response is accurate if the number of data samples for estimating the unknown target channel response goes to infinity. Similarly, the conventional detector for the known the target channel response is the matched filter. We then use the signal-to-noise-ratio gain (SNRG) as a metric to quantify the performance advantage of the time reversal detection scheme over the conventional detector. This SNR gain serves as an asymptotic performance bound for the TRGLRT detector in which the target channel is unknown and needs to be estimated from the measurements.

\section{Time Reversal Detection Problem}

We consider an active multiple antenna system with a pair of antenna arrays $\mathrm{A}$ and $\mathrm{B}$ in a multi-static configuration. The antenna array $\mathrm{A}$ has $P$ elements $A_{1}, \cdots, A_{P}$. The antenna array $\mathrm{B}$ has $N$ elements $B_{1}, \cdots, B_{N}$. It is assumed that both antenna arrays A and B can switch between transmit mode and receive mode. The transmitted signal $s(t)$ is a wideband signal with Fourier representation $S\left(f_{q}\right)$ at frequencies $f_{q}, q=0, \cdots, Q-1$. For simplicity, we assume that the signals transmitted from the antennas $A_{p}, p=1, \cdots, P$, are all identical, i.e., $s_{p}(t)=s(t)$. The total transmission energy is defined as

$$
E_{T}=\frac{1}{Q} \sum_{q=0}^{Q-1}\left\|\mathbf{s}_{A}\left(f_{q}\right)\right\|^{2}=P E_{s}
$$




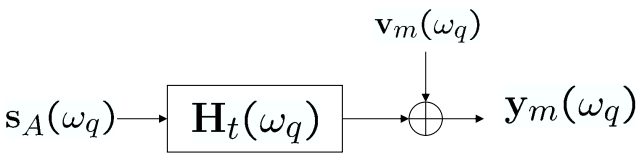

(a). Target monitoring from array $\mathrm{A}$

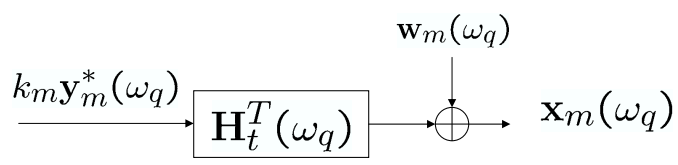

(b). Time reversal retransmission from array B

\section{Figure 1. Simplified Time Reversal Signal Model.}

where $E_{s}=\frac{1}{Q}\left|S\left(f_{q}\right)\right|^{2}$ is the total energy of the probing signal at each antenna, and

$$
\mathbf{s}_{A}\left(f_{q}\right)=\left[S_{1}\left(f_{q}\right), \cdots, S_{P}\left(f_{q}\right)\right]^{T}=S\left(f_{q}\right) \mathbf{1}_{P},
$$

where $\mathbf{1}_{P}$ is the vector with all the elements being 1 .

\subsection{Time Reversal Signal Model}

We introduce the following two channel frequency response matrices to represent the scattering characteristics of the radar target and clutter response. The $N \times P$ clutter channel frequency response matrix $\mathbf{H}_{c}\left(f_{q}\right), q=0, \cdots, Q-$ 1 , is the response of the channel when no target is present; the $(n, p)$-th entry of $\mathbf{H}_{c}\left(f_{q}\right)$ is the channel response between antenna $A_{p}$ and antenna $B_{n}$ at $f_{q}$, i.e.,

$$
\left[\mathbf{H}_{c}\left(f_{q}\right)\right]_{n, p}=h_{c}\left(f_{q} ; B_{n} \leftarrow A_{p}\right), \quad \forall p, n .
$$

The $N \times P$ target channel frequency response $\mathbf{H}_{t}\left(f_{q}\right), q=$ $0, \cdots, Q-1$, is the difference between the channel response when a target is present and the channel response when no target is present. Again, the $(n, p)$-th entry of $\mathbf{H}_{t}\left(f_{q}\right)$ is the target channel response between antenna $A_{p}$ and antenna $B_{n}$, i.e.,

$$
\left[\mathbf{H}_{t}\left(f_{q}\right)\right]_{n, p}=h_{t}\left(f_{q} ; B_{n} \leftarrow A_{p}\right), \quad \forall p, n .
$$

The target channel response $\mathbf{H}_{t}\left(f_{q}\right)$ represents all the changes to $\mathbf{H}_{c}\left(f_{q}\right)$ induced by the presence of the target, which includes the direct echo and the secondary backscatters. Throughout the paper, it is assumed that the clutter channel frequency response $\mathbf{H}_{c}\left(f_{q}\right), \forall q$, is learned and subtracted out from the measurements (see [1] for details). Therefore, it suffices to focus on $\mathbf{H}_{t}\left(f_{q}\right)$. The simplified time reversal signal model is shown in Fig. 1.

For $m$-th snapshot where $m=1, \cdots, M$, we denote by $Y_{m, p, n}\left(f_{q}\right)$ the received signal at antenna $B_{n}$ transmitted from antenna $A_{p}$ at frequency $f_{q}$. We group all the measurements collected at the antennas $B_{n}, n=1, \cdots, N$. This yields an $N$-dimensional signal vector

$$
\mathbf{y}_{m, p}\left(f_{q}\right)=\left[Y_{m, p, 1}\left(f_{q}\right), \cdots, Y_{m, p, N}\left(f_{q}\right)\right]^{T}, \quad \forall p, m .
$$

The signal vector received at antenna $B_{n}$ that is transmitted from antenna $A_{p}$ takes the following form:

$$
\mathbf{y}_{m, p}\left(f_{q}\right)=\mathbf{H}_{t}\left(f_{q}\right) S\left(f_{q}\right) \mathbf{e}_{p}+\mathbf{v}_{m, p}\left(f_{q}\right), \quad \forall p, m,
$$

where $\mathbf{e}_{p}$ is a column vector of zero entries except that its $p$ th element is $1 . \mathbf{v}_{m, p}\left(f_{q}\right)$ is the noise vector at $f_{q}$. The total signal vector received at array B for $m$-th snapshot is

$$
\mathbf{y}_{m}\left(f_{q}\right)=\sum_{p=0}^{P-1} \mathbf{y}_{m, p}\left(f_{q}\right)=\mathbf{H}_{t}\left(f_{q}\right) \mathbf{s}_{A}\left(f_{q}\right)+\mathbf{v}_{m}\left(f_{q}\right),
$$

where the signal vector $\mathbf{s}_{A}\left(f_{q}\right)$ and the complex Gaussian noise vector $\mathbf{v}_{m}\left(f_{q}\right)$ are given as follows:

$$
\mathbf{v}_{m}\left(f_{q}\right)=\left[V_{m, 1}\left(f_{q}\right), \cdots, V_{m, N}\left(f_{q}\right)\right]^{T} .
$$

For $m$ th snapshot, collecting frequency response vectors $\mathbf{y}_{m}\left(f_{q}\right), q=0, \cdots, Q-1$ into an $N Q$-dimensional vector yields

$$
\mathbf{y}_{m}=\left[\mathbf{y}_{m}^{T}\left(\omega_{0}\right), \cdots, \mathbf{y}_{m}^{T}\left(\omega_{Q-1}\right)\right]^{T} .
$$

Next, in the time reversal transmission, each data vector $\mathbf{y}_{m}\left(f_{q}\right)$ is time reversed, power normalized, and retransmitted back into the scattering medium. The $P \times 1$ received signal vector at array $\mathrm{A}$ is

$$
\begin{aligned}
\mathbf{x}_{m}\left(f_{q}\right)= & k_{m} \mathbf{H}_{t}^{T}\left(f_{q}\right) \mathbf{y}_{m}^{*}\left(f_{q}\right)+\mathbf{w}_{m}\left(f_{q}\right), \\
= & k_{m} \mathbf{H}_{t}^{T}\left(f_{q}\right)\left(\mathbf{H}_{t}^{*}\left(f_{q}\right) \mathbf{s}_{A}^{*}\left(f_{q}\right)\right. \\
& \left.+\mathbf{v}_{m}^{*}\left(f_{q}\right)\right)+\mathbf{w}_{m}\left(f_{q}\right) .
\end{aligned}
$$

We notice that in (11) the target channel response becomes $\mathbf{H}^{T}\left(f_{q}\right)$ due to reciprocity of the channel. $\mathbf{w}_{m}$ is the complex Gaussian noise vector

$$
\mathbf{w}_{m}\left(f_{q}\right)=\left[W_{m, 1}\left(f_{q}\right), \cdots, W_{m, P}\left(f_{q}\right)\right]^{T}, \quad \forall p, m .
$$

The scalar $k_{m}$ is the energy normalization factor for each antenna $B_{n}$ and takes the following form:

$$
k_{m}=\sqrt{\frac{\sum_{q=0}^{Q-1}\left\|\mathbf{s}_{A}\left(f_{q}\right)\right\|^{2}}{\sum_{q=0}^{Q-1}\left\|\mathbf{y}_{m}\left(f_{q}\right)\right\|^{2}}}=\sqrt{\frac{Q P E_{s}}{\sum_{q=0}^{Q-1}\left\|\mathbf{y}_{m}\left(f_{q}\right)\right\|^{2}}} .
$$

Furthermore, we define data vectors $\mathbf{x}_{m}, \mathbf{v}_{m}$ and $\mathbf{w}_{m}$ as follows:

$$
\begin{aligned}
\mathbf{x}_{m} & =\left[\mathbf{x}_{m}^{T}\left(\omega_{0}\right), \cdots, \mathbf{x}_{m}^{T}\left(\omega_{Q-1}\right)\right]^{T}, \\
\mathbf{v}_{m} & =\left[\mathbf{v}_{m}^{T}\left(\omega_{0}\right), \cdots, \mathbf{v}_{m}^{T}\left(\omega_{Q-1}\right)\right]^{T}, \\
\mathbf{w}_{m} & =\left[\mathbf{w}_{m}^{T}\left(\omega_{0}\right), \cdots, \mathbf{w}_{m}^{T}\left(\omega_{Q-1}\right)\right]^{T} .
\end{aligned}
$$


Now the time reversal detection problem can be formulated as a binary hypothesis test based upon the concatenated measurements $\left(\mathbf{y}_{m}, \mathbf{x}_{m}\right), \forall m$.

For the conventional detection we use a pair of measurements $\left(\mathbf{y}_{m}, \mathbf{r}_{m}\right)$, where $\mathbf{y}_{m}$ is defined in (9) and

$$
\begin{aligned}
\mathbf{r}_{m}\left(f_{q}\right) & =\beta \mathbf{H}^{T}\left(f_{q}\right) \mathbf{s}_{B}\left(f_{q}\right)+\mathbf{w}_{m}\left(f_{q}\right), \\
\mathbf{r}_{m} & =\left[\mathbf{r}_{m}\left(\omega_{0}\right), \cdots, \mathbf{r}_{m}\left(\omega_{Q-1}\right)\right]^{T} \\
\mathbf{s}_{B}\left(f_{q}\right) & =\left[S_{1}\left(f_{q}\right), \cdots, S_{N}\left(f_{q}\right)\right]^{T} .
\end{aligned}
$$

where the energy normalization factor $\beta$ is defined by

$$
\beta=\sqrt{\frac{\sum_{q=0}^{Q-1}\left\|\mathbf{s}_{A}\left(f_{q}\right)\right\|^{2}}{\sum_{q=0}^{Q-1}\left\|\mathbf{s}_{B}\left(f_{q}\right)\right\|^{2}}}=\sqrt{\frac{P}{N}}
$$

We shall notice that the signal vector $\beta \mathbf{s}_{B}\left(f_{q}\right)$ transmitted by array $\mathrm{B}$ is used to illuminate the medium uniformly. This is contrast to the time reversal detection method where an energy normalized, time-reversed signal $k_{m} \mathbf{y}_{m}^{*}\left(f_{q}\right)$ is transmitted by array B. Using $\left(\mathbf{y}_{m}, \mathbf{x}_{m}\right)$ for time reversal and using $\left(\mathbf{y}_{m}, \mathbf{r}_{m}\right)$ for conventional detection ensures that, for bench-marking purposes, the SNR will be the same in both detection problems.

\subsection{The Detectors}

Let $\mathbf{z}_{m}=\left[\begin{array}{ll}\mathbf{y}_{m}^{T} & \mathbf{x}_{m}^{T}\end{array}\right]^{T}$ and $\mathbf{u}_{m}=\left[\begin{array}{ll}\mathbf{y}_{m}^{T} & \mathbf{r}_{m}^{T}\end{array}\right]^{T}$. We formulate the time reversal binary hypothesis test as follows:

$$
\begin{aligned}
& \mathbb{H}_{1}: \mathbf{z}_{m}=\left[\begin{array}{l}
\mathbf{y}_{t} \\
\mathbf{x}_{t}
\end{array}\right]+\left[\begin{array}{c}
\mathbf{v}_{m} \\
\mathbf{w}_{m} \\
\mathbf{v}_{m} \\
\mathbf{w}_{m}
\end{array}\right], \\
& \mathbb{H}_{0}: \mathbf{z}_{m}=
\end{aligned}
$$

where $\mathbf{x}_{t}\left(f_{q}\right)=k_{m} \mathbf{H}_{t}^{T}\left(f_{q}\right)\left[\mathbf{H}_{t}\left(f_{q}\right) \mathbf{s}_{A}\left(f_{q}\right)+\mathbf{v}_{m}\left(f_{q}\right)\right]^{*}$, and $\mathbf{y}_{t}\left(f_{q}\right)=\mathbf{H}_{t}\left(f_{q}\right) \mathbf{s}_{A}\left(f_{q}\right)$, and

$$
\begin{aligned}
& \mathbf{x}_{t}=\left[\mathbf{x}_{t}^{T}\left(f_{0}\right), \cdots, \mathbf{x}_{t}^{T}\left(f_{Q-1}\right)\right]^{T} \\
& \mathbf{y}_{t}=\left[\mathbf{y}_{t}^{T}\left(f_{0}\right), \cdots, \mathbf{y}_{t}^{T}\left(f_{Q-1}\right)\right]^{T}
\end{aligned}
$$

Similarly, the conventional detection problem can be formulated as below:

$$
\begin{array}{ll}
\mathbb{H}_{1}: & \mathbf{u}_{m}=\left[\begin{array}{c}
\mathbf{y}_{t} \\
\mathbf{r}_{t}
\end{array}\right]+\left[\begin{array}{c}
\mathbf{v}_{m} \\
\mathbf{w}_{m} \\
\mathbf{v}_{m} \\
\mathbf{w}_{m}
\end{array}\right],
\end{array}
$$

where $\mathbf{r}_{t}\left(f_{q}\right)=\beta \mathbf{H}_{t}^{T}\left(f_{q}\right) \mathbf{s}_{B}\left(f_{q}\right)$, and

$$
\mathbf{r}_{t}=\left[\mathbf{r}_{t}^{T}\left(f_{0}\right), \cdots, \mathbf{r}_{t}^{T}\left(f_{Q-1}\right)\right]^{T} .
$$

The optimal detector of (21) is the likelihood ratio of the joint pdfs $p_{1}(\cdot)$ under $\mathbb{H}_{1}$ and $p_{0}(\cdot)$ under $\mathbb{H}_{0}$ of $\left(\mathbf{y}_{m}\left(f_{q}\right), \mathbf{x}_{m}\left(f_{q}\right)\right)$ for a given $m$ (Note that using $M$ snapshots only shifts the receiver operating characteristic curves to the left, but it does not change the performance gap between the asymptotic time reversal detector and the conventional detector. Therefore, it suffices to examine only the $m$-th snapshot.)

$$
\begin{aligned}
\ell_{\text {TR-GLRT(Asym) }}= & \frac{\prod_{q=0}^{Q-1} p_{1}\left(\mathbf{y}_{m}\left(f_{q}\right), \mathbf{x}_{m}\left(f_{q}\right)\right)}{\prod_{q=0}^{Q-1} p_{0}\left(\mathbf{y}_{m}\left(f_{q}\right), \mathbf{x}_{m}\left(f_{q}\right)\right)} \\
= & \prod_{q=0}^{Q-1} \frac{p_{1}\left(\mathbf{x}_{m}\left(f_{q}\right) \mid \mathbf{y}_{m}\left(f_{q}\right)\right)}{p_{0}\left(\mathbf{x}_{m}\left(f_{q}\right) \mid \mathbf{y}_{m}\left(f_{q}\right)\right)} \\
& \prod_{q=0}^{Q-1} \frac{p_{1}\left(\mathbf{y}_{m}\left(f_{q}\right)\right)}{p_{0}\left(\mathbf{y}_{m}\left(f_{q}\right)\right)}
\end{aligned}
$$

The second line in (27) is due to the Bayesian rule. Similarly, the optimal conventional detector of (24) is the likelihood ratio of the joint pdfs of $\left(\mathbf{y}_{m}\left(f_{q}\right), \mathbf{r}_{m}\left(f_{q}\right)\right)$. Since $\mathbf{y}_{m}\left(f_{q}\right)$ and $\mathbf{r}_{m}\left(f_{q}\right)$ are independent, we can break the joint pdf as follows:

$$
\ell_{\mathrm{CDCMF}}=\prod_{q=0}^{Q-1} \frac{p_{1}\left(\mathbf{r}_{m}\left(f_{q}\right)\right)}{p_{0}\left(\mathbf{r}_{m}\left(f_{q}\right)\right)} \prod_{q=0}^{Q-1} \frac{p_{1}\left(\mathbf{y}_{m}\left(f_{q}\right)\right)}{p_{0}\left(\mathbf{y}_{m}\left(f_{q}\right)\right)}
$$

\section{SNR Gain of Time Reversal}

Direct inspection of (27) and (28) reveals that the term $\prod_{q=0}^{Q-1} \frac{p_{1}\left(\mathbf{y}_{m}\left(f_{q}\right)\right)}{p_{0}\left(\mathbf{y}_{m}\left(f_{q}\right)\right)}$ is common to both two detectors. This term does not affect the relative performance of (27) and (28). Therefore, it suffices to examine the remaining terms of each detector for performance comparison. By further examining the statistics of each remaining term, we notice that both of them are complex Gaussian distributed with a non-zero deterministic mean, which implies that the performance comparison can be well characterized by the ratio of the output signal-to-noise-ratio of the corresponding detectors:

$$
\begin{aligned}
d_{\text {TR-GLRT(Asym) }}^{2} & =\frac{\left\|\mathbf{x}_{t}\right\|^{2}}{\sigma_{w}^{2}}=\frac{k_{m}^{2}}{\sigma_{w}^{2}} \sum_{q=0}^{Q-1}\left\|\mathbf{H}_{t}^{T}\left(f_{q}\right) \mathbf{y}_{m}^{*}\left(f_{q}\right)\right\|^{2} \\
d_{\mathrm{CDCMF}}^{2} & =\frac{\left\|\mathbf{r}_{t}\right\|^{2}}{\sigma_{w}^{2}}=\frac{\beta^{2}}{\sigma_{w}^{2}} \sum_{q=0}^{Q-1}\left\|\mathbf{H}_{t}^{T}\left(f_{q}\right) \mathbf{s}_{B}\left(f_{q}\right)\right\|^{2}
\end{aligned}
$$

We then define the ratio of the expectation of these two quantities as the average SNR gain of TR-GLRT(Asym). Given (13), we have

$$
\begin{aligned}
\overline{\mathrm{SNRG}} & \triangleq \frac{E\left\{d_{\text {TR-GLRT(Asym) }}^{2}\right\}}{E\left\{d_{\mathrm{CDCMF}}^{2}\right\}} \\
& =\frac{(N / P) \sum_{q=0}^{Q-1}\left\|\mathbf{s}_{A}\left(f_{q}\right)\right\|^{2}}{\sum_{q=0}^{Q-1}\left\|\mathbf{H}_{t}^{T}\left(f_{q}\right) \mathbf{s}_{B}\left(f_{q}\right)\right\|^{2}} E\left\{\frac{\Phi}{\Upsilon}\right\}
\end{aligned}
$$


where the quantities inside of $E\{\cdot\}$ are

$$
\begin{aligned}
\Phi & =\sum_{q=0}^{Q-1}\left\|\mathbf{H}_{t}^{T}\left(f_{q}\right) \mathbf{H}_{t}^{*}\left(f_{q}\right) \mathbf{s}_{A}^{*}\left(f_{q}\right) \mathbf{H}_{t}^{T}\left(f_{q}\right) \mathbf{v}_{m}^{*}\left(f_{q}\right)\right\|^{2} \\
\Upsilon & =\sum_{q=0}^{Q-1}\left\|\mathbf{H}_{t}\left(f_{q}\right) \mathbf{s}_{A}\left(f_{q}\right)+\mathbf{v}_{m}\left(f_{q}\right)\right\|^{2}
\end{aligned}
$$

To evaluate $\overline{\mathrm{SNRG}}$, the statistics of random variables $\Phi$ and $\Upsilon$ are calculated. First we show that

$E\{\Phi\}=\sum_{q=0}^{Q-1}\left\|\mathbf{H}_{t}\left(f_{q}\right)\right\|^{2} \sigma_{v}^{2}+\sum_{q=0}^{Q-1}\left\|\mathbf{H}_{t}^{T}\left(f_{q}\right) \mathbf{H}_{t}^{*}\left(f_{q}\right) \mathbf{s}_{A}^{*}\left(f_{q}\right)\right\|^{2}$.

Let $t_{k}\left(f_{q}\right)$ denote $k$ th entry of vector $\mathbf{H}_{t}^{T}\left(f_{q}\right) \mathbf{y}_{m}^{*}\left(f_{q}\right)$. Complex Gaussian random variables $V_{m, n}\left(f_{q}\right) \sim$ $\mathcal{C N}\left(0, \sigma_{v}^{2}\right)$, where $\sim$ denotes distribution. We then obtain

$$
\begin{aligned}
t_{k}\left(f_{q}\right)= & \sum_{j=1}^{P} \sum_{i=1}^{N} h_{i k}\left(f_{q}\right) h_{i j}^{*}\left(f_{q}\right) S_{j}^{*}\left(f_{q}\right) \\
& +\sum_{i=1}^{N} h_{i k}\left(f_{q}\right) V_{m, i}^{*}\left(f_{q}\right) \\
\sim & \mathcal{C N}\left(\sum_{j=1}^{P} \sum_{i=1}^{N} h_{i k}\left(f_{q}\right) h_{i j}^{*}\left(f_{q}\right) S_{j}^{*}\left(f_{q}\right),\right. \\
& \left.\sum_{i=1}^{N}\left|h_{i k}\left(f_{q}\right)\right|^{2} \sigma_{v}^{2}\right) \\
\left|t_{k}\left(f_{q}\right)\right|^{2} \sim & \chi_{\sum_{i=1}^{N}\left|h_{i k}\left(f_{q}\right)\right|^{2} \sigma_{v}^{2}}^{2}\left(\mid \sum_{j=1}^{P} \sum_{i=1}^{N}\right. \\
& \left.\left.h_{i k}\left(f_{q}\right) h_{i j}^{*}\left(f_{q}\right) S_{j}^{*}\left(f_{q}\right)\right|^{2}\right) .
\end{aligned}
$$

Therefore, given (31), we have

$$
\begin{aligned}
\Phi & =\sum_{q=0}^{Q-1} \sum_{k=1}^{P}\left|t_{k}\left(f_{q}\right)\right|^{2} \\
\sim & \chi_{\sum_{q=0}^{Q}}^{Q-1} \sum_{k=1}^{P} \sum_{i=1}^{N}\left|h_{i k}\left(f_{q}\right)\right|^{2} \sigma_{v}^{2} \\
& \left(\sum_{q=0}^{Q-1} \sum_{k=1}^{P}\left|\sum_{j=1}^{P} \sum_{i=1}^{N} h_{i k}\left(f_{q}\right) h_{i j}^{*}\left(f_{q}\right) S_{j}^{*}\left(f_{q}\right)\right|^{2}\right) \\
\sim & \chi_{\sum_{q=0}^{Q-1}\left\|\mathbf{H}_{t}\left(f_{q}\right)\right\|^{2} \sigma_{v}^{2}}\left(\sum_{q=0}^{Q-1} \| \mathbf{H}_{t}^{T}\left(f_{q}\right)\right. \\
& \left.\mathbf{H}_{t}^{*}\left(f_{q}\right) \mathbf{s}_{A}^{*}\left(f_{q}\right) \|^{2}\right) .
\end{aligned}
$$

From [2], the mean of a non-central $\chi^{2}-$ distributed random variable is the sum of the degrees of freedom and the noncentral parameter, then (32) follows. Similar to the treatment in deriving the distribution of $\Phi$, the distribution of $\Upsilon$ can be obtained as follows:

$$
\Upsilon \sim \chi_{\sigma_{v}^{2} N Q}^{2}\left(\sum_{q=0}^{Q-1}\left\|\mathbf{H}_{t}\left(f_{q}\right) \mathbf{s}_{A}\left(f_{q}\right)\right\|^{2}\right),
$$

which yields an approximation of the first order inverse moment of the non-central chi-squared distribution, [3],

$$
E\left\{\frac{1}{\Upsilon}\right\} \approx \frac{1}{N Q \sigma_{v}^{2}+\sum_{q=0}^{Q-1}\left\|\mathbf{H}_{t}\left(f_{q}\right) \mathbf{s}_{A}\left(f_{q}\right)\right\|^{2}} .
$$

To obtain a closed form expression of $E\left\{\frac{\Phi}{\Upsilon}\right\}$ we make the following approximation that $\Phi$ and $\Upsilon$ are assumed to be independent random variables, or $E\left\{\frac{\Phi}{\Upsilon}\right\} \approx E\{\Phi\} E\left\{\frac{1}{\Upsilon}\right\}$. Our simulation shows that this is a quite good approximation, especially when $Q$ is large. Plugging (40) and (32) into (30) yields (37).

Next we make some comments regarding this $\overline{\mathrm{SNRG}}$ :

1. If the time reversed signal is noise free, i.e, $\sigma_{v}^{2}=0$ in $\mathbf{y}_{m}$, this gain reduces to the maximal SNR gain for the time reversal method (see [1] for maximal SNR gain with a single antenna pair).

2. When $M_{Y}$ snapshots of $\mathbf{y}_{m}\left(f_{q}\right), m=1, \cdots, M_{Y}$ are used for averaging to reduce the noise variance in time reversed signal, this gain takes the form of (37) except that $\sigma_{v}^{2}$ is replaced by $\sigma_{v}^{2} / M_{y}$ (see Fig. 2 for $M_{y}=20$ and $M_{y}=1$ ).

3. In our development of $\overline{\mathrm{SNRG}}$, it is assumed that the target channel matrix $\mathbf{H}_{t}\left(f_{q}\right)$ is known. Thus this gain represents the asymptotic performance gain of the time reversal GLRT (TR-GLRT) where $\mathbf{H}_{t}\left(f_{q}\right)$ is unknown and needs to be estimated from the (infinite number of data) measurements.

\section{Experiments and Simulations}

A set of scattering channels, with and without the target, in electromagnetic domain with 30 scatterers is measured in a laboratory environment. $Q=51$ uniform frequency samples in $4-6 \mathrm{GHz}$ frequency band are captured. For each antenna array $\mathrm{A}$ and $\mathrm{B}$, a total of 10 positions with inter-element spacing of $10.16 \mathrm{~cm}$ are recorded. A configuration where array $\mathrm{A}$ has 3 antennas at positions with indexes $A=[2,5,9]$ and array $\mathrm{B}$ has 2 antennas at positions with indexes $B=[2,5]$ is chosen as an example. Other choice of array configuration will lead to different detection performance. Fig. 2 depicts the ROC curves for the TR$\mathrm{CMF}$ - the matched filter detector where the time reversed signal is noise free, CDCMF - the conventional matched filter detector, and the TR-GLRT(Asym) - the time reversal detector where noise is considered in the time-reversed signal. The SNR gain of TRCMF over CDCMF stands for the maximal time reversal gain ( $4.0 \mathrm{~dB}$ in our case). The SNR 


$$
\overline{\mathrm{SNRG}} \approx \frac{\sum_{q=0}^{Q-1}\left\|\mathbf{s}_{A}\left(f_{q}\right)\right\|^{2}\left[\sum_{q=0}^{Q-1}\left\|\mathbf{H}_{t}^{T}\left(f_{q}\right) \mathbf{H}_{t}^{*}\left(f_{q}\right) \mathbf{s}_{A}^{*}\left(f_{q}\right)\right\|^{2}+\sigma_{v}^{2} \sum_{q=0}^{Q-1}\left\|\mathbf{H}_{t}\left(f_{q}\right)\right\|^{2}\right]}{(P / N) \sum_{q=0}^{Q-1}\left\|\mathbf{H}_{t}^{T}\left(f_{q}\right) \mathbf{s}_{B}\left(f_{q}\right)\right\|^{2}\left[\sum_{q=0}^{Q-1}\left\|\mathbf{H}_{t}\left(f_{q}\right) \mathbf{s}_{A}\left(f_{q}\right)\right\|^{2}+\sigma_{v}^{2} N Q\right]}
$$

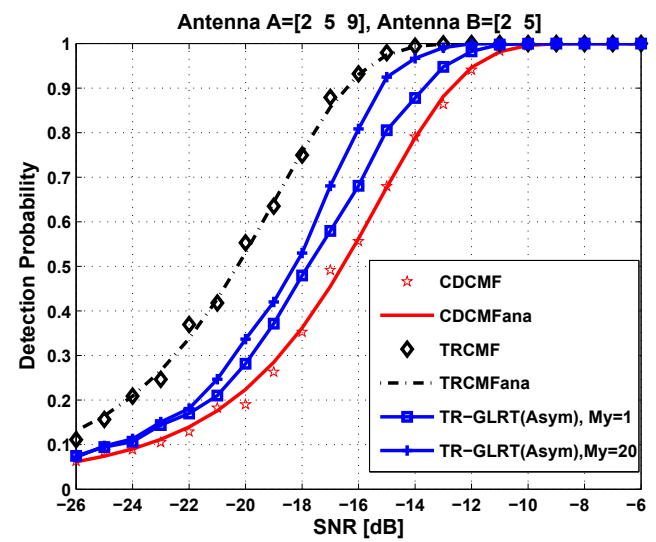

Figure 2. ROC curve for TRCMF-TR channel matched filter when the time reversed signal is noise free, versus CDCMF-conventional matched filter, and TR-GLRT(Asym)-where the time reversed signal contains noise. $\sigma_{v}^{2}=$ $\sigma_{w}^{2}$. False alarm rate $P_{\mathrm{FA}}=0.01$.

gain of TR-GLRT(Asym) at detection rate of 0.8 is about $2.2 \mathrm{~dB}$ when $M_{y}=20$, and $1.2 \mathrm{~dB}$ when $M_{y}=1$. The loss is due to the contamination of the noise in the time reversed signal. The $\overline{\mathrm{SNRG}}$ of TR-GLRT(Asym) is plotted in Fig. 3. The simulation results match well the analytical results. For example, at SNR of $-16 \mathrm{~dB}$ in Fig. 2, we observe a $2.2 \mathrm{~dB}$ gain for TR-GLRT(Asym) when $M_{y}=20$ over CDCMF. The predicted $\overline{\mathrm{SNRG}}$ at SNR of $-16 \mathrm{~dB}$ reads 2.2 dB. Fig. 4 depicts the ROC curve for TR-GLRT vs. energy detector. The SNR gain at $P_{D}=0.8$ reads as $0.8 \mathrm{~dB}$, which is a little less than the asymptotic SNR gain of $1.2 \mathrm{~dB}$.

\section{Conclusion}

Time reversal takes advantage of the rich scattering environment. The richer the scattering, the higher the time reversal performance gain relative to the conventional detection. However, the noise has an adverse impact on the time reversal detection. In this paper, we derived the analytical expression of the time reversal asymptotic SNR gain over the conventional detection. We verified our analysis using the experimentally obtained scattering channel measurements in electromagnetic domain. The analysis and experimental results demonstrate that a rich scattering and quiet environment is preferred for time reversal.

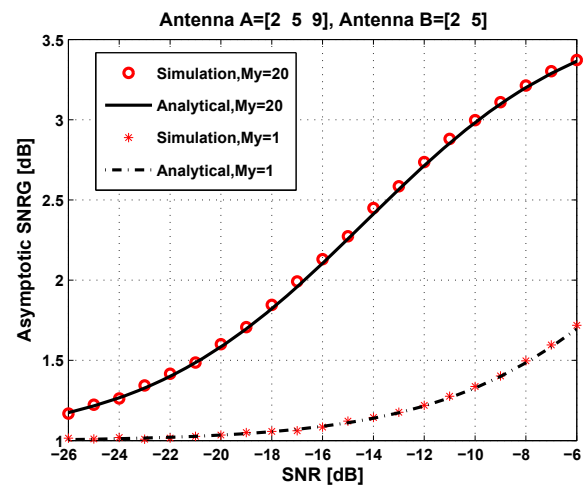

Figure 3. SNRG: simulation vs. analytical results.

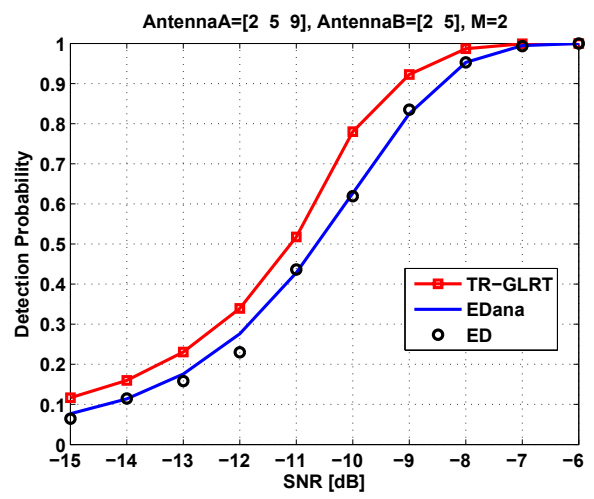

Figure 4. ROC curve for TR-GLRT vs. energy detector (ED). $\sigma_{v}^{2}=\sigma_{w}^{2}$. $P_{\mathrm{FA}}=0.01$.

\section{References}

[1] J. M. F. Moura and Y. Jin, "Detection by time reversal: single antenna," IEEE Transactions on Signal Processing, December 2006, to appear.

[2] N. L. Johnson and S. Kotz, Continuous Univariate Distributions-2nd edition. New York, NY: John Wiley \& Sons, Inc., 1970.

[3] T. Fujioka, "Asymptotic approximations of the inverse moment of the noncentral chi-squared variable," $J$. Japan Statistics Society, vol. 31, no. 1, pp. 99-109, 2001. 